\title{
COMBINATORIAL CONSTRUCTIONS FOR INTEGRALS OVER NORMALLY DISTRIBUTED RANDOM MATRICES
}

\author{
I. P. GOULDEN AND D. M. JACKSON \\ (Communicated by Jeffry N. Kahn)
}

\begin{abstract}
Recent results of Hanlon, Stanley, and Stembridge give the expected values of certain functions of matrices of normal variables in the real and complex cases. They point out that in both cases the results are equivalent to combinatorial results and suggest further that these results may have purely combinatorial proofs, in this way avoiding the use of the theory of spherical functions. Such proofs are given in this paper. In the complex case we use the familiar cycle decomposition for permutations. In the real case we introduce an analogous decomposition into cyclically ordered sequences, called chains, which makes the real and complex cases strikingly similar.
\end{abstract}

\section{INTRODUCTION}

If $\lambda_{1}+\cdots+\lambda_{n}=k$ for nonnegative integers $\lambda_{1} \geq \cdots \geq \lambda_{n}>0$, then $\lambda=\left(\lambda_{1}, \ldots, \lambda_{n}\right)$ is a partition of $k$ and we write $\lambda \vdash k$. Let $a=\left(a_{1}, a_{2}, \ldots\right)$ and $b=\left(b_{1}, b_{2}, \ldots\right)$ where $a_{1}, a_{2}, \ldots$ and $b_{1}, b_{2}, \ldots$ are indeterminates. Let $p_{k}(a)=\sum_{j \geq 1} a_{j}^{k}, k \geq 1$, a power sum symmetric function, and $p_{\lambda}(a)=$ $p_{\lambda_{1}}(a) \cdots p_{\lambda_{n}}(a)$. We also use $p_{\lambda}(\mathbf{A})$, where $p_{\lambda_{i}}(\mathbf{A})$ denotes $\operatorname{trace}\left(\mathbf{A}^{\lambda_{i}}\right)$ for a square matrix $\mathbf{A}$.

Hanlon, Stanley, and Stembridge [2, Theorem 2.3] prove that, for $\lambda \vdash k$,

$$
\mathscr{E}_{\mathbf{U}}\left(p_{\lambda}\left(\mathbf{A U B U}^{*}\right)\right)=\sum_{\mu, \nu \vdash k} c_{\mu, \nu}^{\lambda} p_{\mu}(a) p_{\nu}(b),
$$

where the expectation is over $n \times n$ matrices $\mathrm{U}$ whose entries are independent standard normal random complex variables and A, B are fixed, but arbitrary, Hermitian complex matrices. On the right-hand side, $a_{1}, \ldots, a_{n}, b_{1}, \ldots, b_{n}$ are the eigenvalues of $\mathbf{A}, \mathbf{B}$ with the remaining $a$ 's, $b$ 's set equal to zero and $c_{\mu, \nu}^{\lambda}$ is the connection coefficient for the class algebra in $\mathbb{C G}_{k}$, the group algebra of the symmetric group $\mathfrak{G}_{k}$ on $k$ symbols.

The main result of [2] (Theorem 3.5) is the analogue of the above result in the real case and states that, for $\lambda \vdash k$,

$$
\mathscr{E}_{\mathbf{U}}\left(p_{\lambda}\left(\mathbf{A U B U}^{T}\right)\right)=\frac{1}{\left|\mathscr{B}_{k}\right|} \sum_{\mu, \nu \vdash k} d_{\mu, \nu}^{\lambda} p_{\mu}(a) p_{\nu}(b),
$$

Received by the editors March 4, 1993 and, in revised form, June 25, 1993.

1991 Mathematics Subject Classification. Primary 05E05, 15A52, $05 A 15$.

Research supported by grants from NSERC. 
where the expectation is over $n \times n$ matrices $\mathrm{U}$ whose elements are independent standard normal random real variables and $\mathbf{A}, \mathbf{B}$ are fixed, but arbitrary, real symmetric matrices. In this case, $d_{\mu, \nu}^{\lambda}$ is the connection coefficient for the Hecke algebra of the Gel'fand pair $\left(\mathfrak{G}_{2 k}, \mathscr{B}_{k}\right)$, where $\mathscr{B}_{k}$ is the hyperoctahedral group.

The proofs given in [2] use the theory of spherical functions. However, alternative evaluations of these expected values (the left-hand sides) as combinatorial sums are also given there, leading those authors to suggest that (1) and (2) may have purely combinatorial proofs.

The following definitions are needed in the description of these sums, which are given below as (3) and (4). Let $\mathscr{W}_{k}$ be the set of all words of length $2 k$ in the elements of $a$ and $b$, starting with an element of $a$ and alternating thereafter between $b$ and $a$ (so that they end with an element of $b$ ). For a partition $\lambda$, let $\mathscr{W}_{\lambda}=\mathscr{W}_{\lambda_{1}} \times \cdots \times \mathscr{W}_{\lambda_{n}}$. For $w=\left(w_{1}, \ldots, w_{n}\right) \in \mathscr{W}_{\lambda}$, let $\psi(w)$ be the commutative image of $w$ (so it is a product of all the $a_{i}$ 's and $b_{j}$ 's in $\left.w_{1}, \ldots, w_{n}\right)$. Let $m_{i, j}(w)$ be the number of times $a_{i} b_{j}$ appears consecutively as a subword among $w_{1}, \ldots, w_{n}$, and let $m_{i, j}^{\prime}(w)$ be the number of times $b_{j} a_{i}$ appears consecutively as a subword among $w_{1}, \ldots, w_{n}$, with the convention that the last letter of each $w_{l}$ is followed by its first letter (so from this point of view the $w_{l}$ are circular words).

Let $\mathscr{W}_{\lambda}^{\prime}$ consist of the words $w \in \mathscr{W}_{\lambda}$ such that $m_{i, j}(w)=m_{i, j}^{\prime}(w)$ for all $i, j \geq 1$. Let $\mathscr{W}_{\lambda}^{\prime \prime}$ consist of the words $w \in \mathscr{W}_{\lambda}$ such that $m_{i, j}(w)+m_{i, j}^{\prime}(w)=$ $\eta_{i, j}(w)$ is even for all $i, j \geq 1$.

The alternative evaluation [2, Proposition 6.2] of the left-hand side of (1) is

$$
\sum_{w \in \mathscr{W}_{i}^{\prime}} \psi(w) \prod_{i, j \geq 1} m_{i, j}(w) !
$$

for which the only integration required is $\mathscr{E}\left(u^{r} \bar{u}^{s}\right)=r ! \delta_{r, s}$, where $u$ is a standard normal complex variable. Similarly, the alternative evaluation [2, Proposition 6.2] of the left-hand side of (2) is

$$
\sum_{w \in \mathscr{W}_{\lambda}^{\prime \prime}} \psi(w) \prod_{i, j \geq 1}\left(\eta_{i, j}(w)-1\right) ! !
$$

for which the only integration required is $\mathscr{E}\left(u^{2 r}\right)=(2 r-1) ! !$, where $(2 r-1) ! !$ denotes $(2 r) ! / 2^{r} r$ ! , and where $u$ is a standard normal real variable.

Hanlon, Stanley, and Stembridge's [2] suggestion is that combinatorial proofs (avoiding spherical functions) of (1) and (2) would be obtained if we could establish combinatorially that (3) is equal to the right-hand side of (1) in the complex case and that (4) is equal to the right-hand side of (2) in the real case, for sets of indeterminates $a$ and $b$.

Such a combinatorial proof is given in [2] for the complex case with singlepart partitions. In this paper, we provide combinatorial proofs for general $\lambda$ in the complex and real cases.

The result for the complex case is given in Theorem 2.1 and, for the real case, in Theorem 3.1. These theorems are shown directly by combinatorial constructions applied to two natural combinatorial structures associated with 
permutations. In the complex case, the structure is the familiar disjoint cycle decomposition. In the real case we introduce a new object, called a chain, for which there is an analogous disjoint decomposition. It is sufficient to work with the representations of these structures as linearized circular sequences rather than with the richer structure of faces of embeddings of graphs. The latter was used in [1] for the determination of certain connection coefficients and then as the basis for the proof of the complex single-part case cited above.

The proofs are presented in a parallel way to show their similarity at the combinatorial level. It is hoped that the constructions may find further application.

For further details on the background to the question addressed here, and for details of the integration theory, the reader is referred to [2].

\section{THE COMPLEX CASE}

For a permutation $\sigma \in \mathfrak{G}_{k}$, let $\tau(\sigma)$, called the cycle-type of $\sigma$, be the partition of $k$ giving the lengths of the cycles in the disjoint cycle decomposition of $\sigma$. In this paper we adopt the convention that cycles are listed in weakly decreasing order of lengths and, within those of the same length, in increasing order of smallest element. The elements on each cycle are listed in the order in which they appear around the cycle, with the smallest element first, and enclosed in round brackets. Let $C_{\lambda}$ be the sum in the group algebra of $\mathfrak{G}_{k}$ of all permutations with cycle-type $\lambda$, for $\lambda$ a partition of $k$. The $\mathbb{C}$-span of the $C_{\mu}$ 's generates the class algebra of the group algebra.

We now give the combinatorial theorem of Hanlon, Stanley, and Stembridge [2] arising from their expected value in the complex case. Their theorem has been rewritten using the fact that the connection coefficients $c_{\mu, \nu}^{\lambda}$ are given by $\mathrm{C}_{\mu} \mathrm{C}_{\nu}=\sum_{\lambda} c_{\mu, \nu}^{\lambda} \mathrm{C}_{\lambda}$ and that the $\mathrm{C}_{\nu}$ commute. Combinatorially, the connection coefficient $c_{\mu, \nu}^{\lambda}$ is the number of ways $\left(e_{1}, e_{2}\right)$ in which an arbitrary element with cycle-type $\lambda$ can be written as $e_{1} e_{2}$, where $e_{1}$ has cycle-type $\mu$ and $e_{2}$ has cycle-type $\nu$. The proof is bijective, involving a construction on words in $\mathscr{W}_{\lambda}^{\prime}$. A brief numerical example is carried along to illuminate certain points of the exposition.

\section{Theorem 2.1.}

$$
\left(\sum_{\nu \vdash k} C_{\nu} p_{\nu}(b)\right)\left(\sum_{\mu \vdash k} C_{\mu} p_{\mu}(a)\right)=\sum_{\lambda \vdash k} C_{\lambda} \sum_{w \in \mathscr{W}_{\lambda}^{\prime}} \psi(w) \prod_{i, j \geq 1} m_{i, j}(w) ! .
$$

Proof. First we give the left-hand side of the result a combinatorial interpretation as follows. For $\rho \in \mathfrak{G}_{k}$, a cycle a-labeling $\alpha$ of $\rho$ is a mapping from the cycles in the disjoint cycle decomposition of $\rho$ to the elements of $a$. In such a mapping, we say that a cycle is a-labeled (or labeled where the context permits) by the element of $a$ to which it is mapped. Let $\operatorname{wt}(\alpha)=\prod_{i \geq 1} a_{i}^{f_{i}}$ where $f_{i}$ is the total number of elements on cycles labeled $a_{i}$ in $\alpha$. Then clearly $\sum_{\alpha} \mathrm{wt}(\alpha)=p_{\tau(\rho)}(a)$, where the sum is over all cycle $a$-labelings $\alpha$ of $\rho$, and thus

$$
\sum_{\rho \in \mathfrak{B}_{k}} \sum_{\alpha} \rho \mathrm{wt}(\alpha)=\sum_{\mu \vdash k} \mathrm{C}_{\mu} p_{\mu}(a)
$$


This means that the left-hand side of the result is the generating function

$$
\sum_{\sigma, \rho \in \mathfrak{B}_{k}} \sum_{\beta, \alpha} \sigma \rho \mathrm{wt}(\beta) \mathrm{wt}(\alpha)
$$

where $\beta$ is summed over all cycle $b$-labelings of $\sigma$.

For the right-hand side, let $\mathscr{P}(w)$ be the set of infinite-dimensional matrices indexed by the positive integers, whose $(i, j)$-entry is some permutation of $\left\{1, \ldots, m_{i, j}(w)\right\}$, for $i, j \geq 1$. Thus $|\mathscr{P}(w)|=\prod_{i, j \geq 1} m_{i, j}(w)$ !, so the right-hand side of the result is the generating function

$$
\sum_{\gamma \in \mathfrak{G}_{k}} \sum_{w \in \mathscr{W}_{\tau(\gamma)}^{\prime}} \gamma \psi(w)|\mathscr{P}(w)| .
$$

We now give a combinatorial proof of the result by comparing the generating functions (5) and (6), and by considering each pair $\sigma, \rho \in \mathfrak{G}_{k}$. All we need, for each cycle $b$-labeling $\beta$ of $\sigma$ and cycle $a$-labeling $\alpha$ of $\rho$, is to construct $\gamma \in \mathfrak{G}_{k}, w \in \mathscr{W}_{\tau(\gamma)}^{\prime}$, and $\mathbf{P} \in \mathscr{P}(w)$ such that $\sigma \rho=\gamma$ and $\operatorname{wt}(\beta) \operatorname{wt}(\alpha)=\psi(w)$ and in such a way that this construction is reversible. The three quantities are constructed as follows.

(i) For $\gamma$ : Compute the product $\sigma \rho$ to obtain $\gamma$.

(ii) For $w$ : Let the cycles of $\gamma$, in the order specified by the above convention, have lengths $\lambda_{1}, \ldots, \lambda_{n}$. For each $j=1, \ldots, n$, construct a word $u_{j}$ of length $4 \lambda_{j}$ by concatenating $\lambda_{j}$ strings of length four where the $l$ th such string is

$$
g a_{d} \rho(g) b_{h},
$$

in which $g$ is the $l$ th element on the $j$ th cycle of $\gamma, a_{d}$ is the label of the cycle containing $g$ in $\alpha$, and $b_{h}$ is the label of the cycle containing $\rho(g)$ in $\beta$.

For example, with $k=10$ and

$$
\sigma=(1285103)(496)(7), \quad \rho=(152)(6710)(39)(48),
$$

let

$$
\beta=(1285103)_{b_{1}}(496)_{b_{2}}(7)_{b_{1}} \text { and } \alpha=(152)_{a_{1}}(6710)_{a_{3}}(39)_{a_{2}}(48)_{a_{2}}
$$

where the subscript on a cycle is its label. Thus $\operatorname{wt}(\beta)=b_{1}^{7} b_{2}^{3}$ and $\operatorname{wt}(\alpha)=$ $a_{1}^{3} a_{2}^{4} a_{3}^{3}$. Then $\gamma=(1104589)(367)(2)$, so we construct

$$
\begin{aligned}
& u_{1}=1 a_{1} 5 b_{1} 10 a_{3} 6 b_{2} 4 a_{2} 8 b_{1} 5 a_{1} 2 b_{1} 8 a_{2} 4 b_{2} 9 a_{2} 3 b_{1}, \\
& u_{2}=3 a_{2} 9 b_{2} 6 a_{3} 7 b_{1} 7 a_{3} 10 b_{1}, \\
& u_{3}=2 a_{1} 1 b_{1} .
\end{aligned}
$$

We obtain $w=\left(w_{1}, \ldots, w_{n}\right) \in \mathscr{W}_{\lambda}$ by retaining in $u=\left(u_{1}, \ldots, u_{n}\right)$ only the $a$ 's and $b$ 's, in place. (Thus we immediately have $\operatorname{wt}(\beta) \operatorname{wt}(\alpha)=\psi(w)$.) We now show that $w$ is in $\mathscr{W}_{\lambda}^{\prime}$. For $i, j \geq 1$, let $\mathscr{D}_{i, j}$ be the set of elements of $\{1, \ldots, k\}$ on a cycle labeled $a_{i}$ in $\alpha$ and on a cycle labeled $b_{j}$ in $\beta$. An occurrence of $a_{i} b_{j}$ as a subword in some $w_{l}$ corresponds to an occurrence of $a_{i} t b_{j}$ as a subword in $u_{l}$, where $t \in \mathscr{D}_{i, j}$, so $m_{i, j}(w)=\left|\mathscr{D}_{i, j}\right|$. Similarly, an occurrence of $b_{j} a_{i}$ as a subword in some $w_{l}$ corresponds to an occurrence of $b_{j} t a_{i}$ as a subword in $u_{l}$, where $t \in \mathscr{D}_{i, j}$ (where the $u_{l}$ are circular when appropriate, to match the $\left.w_{l}\right)$, so $m_{i, j}^{\prime}(w)=\left|\mathscr{D}_{i, j}\right|$. Thus, $m_{i, j}(w)=m_{i, j}^{\prime}(w)$ for $i, j \geq 1$, so $w \in \mathscr{W}_{\lambda}^{\prime}$ with $\lambda=\tau(\gamma)$. 
(iii) For $\mathbf{P}$ : Finally, we obtain $\mathbf{P}$ as the matrix of permutations whose $(i, j)$ entry, $i, j \geq 1$, is the permuted order in which the elements of $\mathscr{D}_{i, j}$ appear from left to right in the third positions $(\bmod 4)$ in $u_{1}$, followed by $u_{2}, \ldots, u_{n}$. Since $\left|\mathscr{D}_{i, j}\right|=m_{i, j}(w)$ and each element of $\{1, \ldots, k\}$ occurs exactly once in the third positions $(\bmod 4)$, then $\mathbf{P} \in \mathscr{P}(w)$ and the construction is complete.

To conclude the above example, we obtain

$$
\begin{aligned}
& w_{1}=a_{1} b_{1} a_{3} b_{2} a_{2} b_{1} a_{1} b_{1} a_{2} b_{2} a_{2} b_{1}, \\
& w_{2}=a_{2} b_{2} a_{3} b_{1} a_{3} b_{1}, \\
& w_{3}=a_{1} b_{1}
\end{aligned}
$$

so

$$
\left[\mathscr{D}_{i, j}\right]=\left[\begin{array}{cccc}
\{1,2,5\} & \varnothing & \varnothing & \cdots \\
\{3,8\} & \{4,9\} & \varnothing & \cdots \\
\{7,10\} & \{6\} & \varnothing & \cdots \\
\varnothing & \varnothing & \varnothing & \cdots \\
\vdots & \vdots & \vdots & \ddots
\end{array}\right] \quad \text { and } \quad \mathbf{P}=\left[\begin{array}{cccc}
321 & \varnothing & \varnothing & \cdots \\
21 & 12 & \varnothing & \cdots \\
12 & 1 & \varnothing & \cdots \\
\varnothing & \varnothing & \varnothing & \cdots \\
\vdots & \vdots & \vdots & \ddots
\end{array}\right]
$$

where each entry of $\mathbf{P}$ is the second row of the two-line representation of the corresponding permutation.

To reverse the construction, we start with $\gamma, w, \mathbf{P}$ and proceed to determine $u$. The second and fourth positions $(\bmod 4)$ of $u$ come immediately from $w$. The first positions $(\bmod 4)$ come immediately from the successive entries in the cycles of $\gamma$. We now find the $\mathscr{D}_{i, j}$ by analyzing substrings $b_{j} t a_{i}$ (which involve only positions 1,2 , and $4(\bmod 4))$. The third positions $(\bmod 4)$ now follow by applying, for $i, j \geq 1$, the $(i, j)$-entry of $\mathbf{P}$ to $\mathscr{D}_{i, j}$, thereby specifying, in order, the entries between $a_{i}$ in the second positions $(\bmod 4)$ and $b_{j}$ in the fourth positions $(\bmod 4)$. Clearly, $u$ uniquely determines $\rho, \sigma, \alpha, \beta$.

\section{THe ReAl CASE}

Let $\mathscr{A}_{k}=\{\{1, \widehat{1}\}, \ldots,\{k, \hat{k}\}\}$. In this section, we now use $\mathfrak{G}_{2 k}$ to denote the symmetric group acting on $\{1, \hat{1}, \ldots, k, \hat{k}\}$, whose elements are ordered by $1 \prec \hat{1} \prec \cdots \prec k \prec \hat{k}$. For $\sigma \in \mathfrak{G}_{2 k}$, a chain is an ordered list of an even number of ordered pairs $\left(\left(i_{1}, j_{1}\right) \cdots\left(i_{2 m}, j_{2 m}\right)\right)$ such that $j_{s}=\sigma\left(i_{s}\right), s=$ $1, \ldots, 2 m$, with left-links $\left\{i_{2 t-1}, i_{2 t}\right\} \in \mathscr{A}_{k}$, and with right-links $\left\{j_{2 t}, j_{2 t+1}\right\} \in$ $\mathscr{A}_{k}, t=1, \ldots, m$ (where $i_{2 m+1}=i_{1}$, so these are interpreted circularly) and $i_{1}$ is the smallest of $i_{1}, \ldots, i_{2 m}$. The $i$ 's are called the left-elements of the chain and the $j$ 's are called the right-elements of the chain (thus, $i_{1}$ is the smallest left-element on the chain). The length of a chain is the number of ordered pairs it contains.

The permutation $\sigma$ is completely specified by its set of chains, which form the chain decomposition $c(\sigma)$ of $\sigma$. We adopt the convention that in $c(\sigma)$ the chains are written in weakly decreasing order by length and, for those of the same length, in increasing order of smallest left-element. The $t$ th chain in this decomposition has $2 \lambda_{t}$ ordered pairs for $t=1, \ldots, n$ for some $\lambda=$ $\left(\lambda_{1}, \ldots, \lambda_{n}\right) \vdash k$ and has chain-type denoted by $\kappa(\sigma)=\lambda$. 
For example, with $k=5, \sigma=(1 \widehat{5} 4 \widehat{3} 2)(\hat{1} \widehat{2} 35 \widehat{4})$ has chain decomposition

$$
c(\sigma)=((1, \widehat{5})(\hat{1}, \widehat{2})(\widehat{3}, 2)(3,5))((2,1)(\widehat{2}, 3)(4, \widehat{3})(\widehat{4}, \widehat{1}))((5, \widehat{4})(\widehat{5}, 4))
$$

and chain-type $\kappa(\sigma)=(2,2,1)$.

Let $\mathrm{K}_{\lambda}$ be the sum in the group algebra of $\mathfrak{G}_{2 k}$ of all permutations with chain-type $\lambda$. Certain algebraic properties of the hyperoctahedral group are needed for discussing $\mathrm{K}_{\lambda}$, and we establish these combinatorially. The hyperoctahedral group $\mathscr{B}_{k}$ is isomorphic to the wreath product $\mathfrak{G}_{2} \backslash \mathfrak{G}_{k}$ and can be embedded in $\mathfrak{G}_{2 k}$ as the centraliser subgroup $\left\{\sigma \in \mathfrak{G}_{2 k}: \sigma \delta=\delta \sigma\right\}$, where $\delta$ has disjoint cycle decomposition $(1 \hat{1}) \cdots(k \widehat{k})$. Thus $\mathscr{B}_{k}$ is the automorphism group of $\mathscr{A}_{k}$ and $\left|\mathscr{B}_{k}\right|=2^{k} k$ ! For $\delta_{1}, \delta_{2} \in \mathscr{B}_{k}$ and $\sigma \in \mathfrak{G}_{2 k}$, the chain decomposition of $\delta_{1} \sigma \delta_{2}$ can be obtained from the chain decomposition of $\sigma$ by applying $\delta_{1}$ to the right-links of $\sigma$ and $\delta_{2}^{-1}$ to the left-links of $\sigma$. Thus $\kappa\left(\delta_{1} \sigma \delta_{2}\right)=\kappa(\sigma)$, so the $\mathrm{K}_{\lambda}$ are precisely the double cosets of $\mathscr{B}_{k}$ in $\mathfrak{G}_{2 k}$. The $\mathbb{C}$-span of $\left\{\mathrm{K}_{\lambda}: \lambda \vdash k\right\}$ generates a commutative subalgebra of $\mathbb{C G}_{2 k}$ called the Hecke algebra of the Gel'fand pair $\left(\mathfrak{G}_{2 k}, \mathscr{B}_{k}\right)$. For further discussion, see [2].

A matching on a set of even cardinality is a (set) partition of the set into disjoint unordered pairs. For example, $\mathscr{A}_{k}$ is a matching. The number of matchings on a $2 m$-set is $(2 m-1) ! !$.

We now give the combinatorial theorem of Hanlon, Stanley, and Stembridge [2] arising from their expected value in the real case. Their theorem has been rewritten using the fact that the connection coefficients $d_{\mu, \nu}^{\lambda}$ are given by $\mathrm{K}_{\mu} \mathrm{K}_{\nu}=\sum_{\lambda} d_{\mu, \nu}^{\lambda} \mathrm{K}_{\lambda}$ and that the $\mathrm{K}_{\nu}$ commute. Combinatorially, the connection coefficient $d_{\mu, \nu}^{\lambda}$ is the number of ways $\left(e_{1}, e_{2}\right)$ in which an arbitrary element with chain-type $\lambda$ can be written as $e_{1} e_{2}$, where $e_{1}$ has chain-type $\mu$ and $e_{2}$ has chain-type $\nu$. The proof is bijective, involving a construction on words in $\mathscr{W}_{\lambda}^{\prime \prime}$.

Theorem 3.1.

$$
\left(\sum_{\nu \vdash k} \mathrm{~K}_{\nu} p_{\nu}(b)\right)\left(\sum_{\mu \vdash k} \mathrm{~K}_{\mu} p_{\mu}(a)\right)=\left|\mathscr{B}_{k}\right| \sum_{\lambda \vdash k} \mathrm{~K}_{\lambda} \sum_{w \in \mathscr{W}_{\lambda}^{\prime \prime}} \psi(w) \prod_{i, j \geq 1}\left(\eta_{i, j}(w)-1\right) ! ! .
$$

Proof. We begin with a combinatorial interpretation of the left-hand side. For $\rho \in \mathfrak{G}_{2 k}$, a chain a-labeling $\alpha$ of $\rho$ is a mapping from the chains in the chain decomposition of $\rho$ to the elements of $a$. In such a mapping, we say that a chain is a-labeled (or labeled where the context permits) by the element of $a$ to which it is mapped. Let $\mathrm{wt}(\alpha)=\prod_{i \geq 1} a_{i}^{f_{i}}$ where $f_{i}$ is half the total number of ordered pairs on chains labeled $a_{i}$ in $\alpha$. Then clearly $\sum_{\alpha} \mathrm{wt}(\alpha)=p_{\kappa(\rho)}(a)$, where the sum is over all chain $a$-labelings $\alpha$ of $\rho$, and thus

$$
\sum_{\rho \in \mathfrak{G}_{2 k}} \sum_{\alpha} \rho \mathrm{wt}(\alpha)=\sum_{\mu \vdash k} \mathrm{~K}_{\mu} p_{\mu}(a) .
$$

This means that the left-hand side of the result is the generating function

$$
\sum_{\sigma, \rho \in \mathfrak{G}_{2 k}} \sum_{\beta, \alpha} \sigma \rho \mathrm{wt}(\beta) \mathrm{wt}(\alpha),
$$

where $\beta$ is summed over all chain $b$-labelings of $\sigma$. 
For the right-hand side, let $\mathscr{M}(w)$ be the set of all infinite-dimensional matrices indexed by the positive integers, whose $(i, j)$-entry is some matching on $\left\{1, \ldots, \eta_{i, j}(w)\right\}$, for $i, j \geq 1$. Thus $|\mathscr{M}(w)|=\prod_{i, j \geq 1}\left(\eta_{i, j}(w)-1\right) ! !$, so the right-hand side of the result is the generating function

$$
\left|\mathscr{B}_{k}\right| \sum_{\gamma \in \mathfrak{B}_{2 k}} \sum_{w \in \mathscr{W}_{\kappa(\gamma)}^{\prime \prime}} \gamma \psi(w)|\mathscr{M}(w)|
$$

We now give a combinatorial proof of the result by comparing (7) and (8) and considering each pair $\sigma, \rho \in \mathfrak{G}_{2 k}$. All we need, for each chain $b$-labeling $\beta$ of $\sigma$ and chain $a$-labeling $\alpha$ of $\rho$, is to construct $d \in \mathscr{B}_{k}, \gamma \in \mathfrak{G}_{2 k}, w \in \mathscr{W}_{\kappa(\gamma)}^{\prime \prime}$ and $\mathbf{M} \in \mathscr{M}(w)$ such that $\sigma \rho=\gamma$ and $\operatorname{wt}(\beta) \mathrm{wt}(\alpha)=\psi(w)$ and in such a way that this construction is reversible. The four quantities are constructed as follows.

(i) For $\gamma$ : Compute the product $\sigma \rho$ to obtain $\gamma$.

(ii) For $w$ : Let the chains of $\gamma$, in the order specified by the above convention, have lengths $2 \lambda_{1}, \ldots, 2 \lambda_{n}$. For each $j=1, \ldots, n$, construct a word $v_{j}$ of length $4 \lambda_{j}$ by concatenating $\lambda_{j}$ strings of length four, where the $l$ th such string is

$$
g a_{d} g^{\prime} b_{h}
$$

in which $g=(s, \rho(s), t), g^{\prime}=\left(s^{\prime}, \rho\left(s^{\prime}\right), t^{\prime}\right)$ where $(s, t)$ and $\left(s^{\prime}, t^{\prime}\right)$ are the $(2 l-1)$ st and $2 l$ th ordered pairs on the $j$ th chain of $\gamma, a_{d}$ is the label of the chain in $\alpha$ containing $s$ (and $s^{\prime}$ ) as a left-element, and $b_{h}$ is the label of the chain in $\beta$ containing $t^{\prime}$ as a right-element. let

For example, with $k=8$ and $\sigma=(\hat{1} 23 \hat{2} 4 \widehat{3})(1)(\widehat{4}), \rho=(1 \hat{1} 42 \widehat{3} \widehat{4})(\widehat{2} 3)$,

$$
\begin{aligned}
& \beta=((1,1)(\hat{1}, 2)(3, \widehat{2})(\widehat{3}, \hat{1}))_{b_{2}}((2,3)(\widehat{2}, 4)(\widehat{4}, \widehat{4})(4, \widehat{3}))_{b_{1}}, \\
& \alpha=((1, \hat{1})(\widehat{1}, 4)(\widehat{3}, \widehat{4})(3, \hat{2})(4,2)(\widehat{4}, 1))_{a_{1}}((2, \widehat{3})(\widehat{2}, 3))_{a_{2}}
\end{aligned}
$$

where the subscript on a chain is its label. Thus $\operatorname{wt}(\beta)=b_{1}^{2} b_{2}^{2}$ and $\operatorname{wt}(\alpha)=$ $a_{1}^{3} a_{2}$. Then $\gamma=(12 \hat{1} \hat{3} \hat{4})(34)(\widehat{2})$, so

$$
c(\gamma)=((1,2)(\widehat{1}, \widehat{3})(4,3)(\widehat{4}, 1)(2, \widehat{1})(\widehat{2}, \widehat{2}))((3,4)(\widehat{3}, \widehat{4}))
$$

and we construct

$$
\begin{aligned}
& v_{1}=(1, \widehat{1}, 2) a_{1}(\hat{1}, 4, \widehat{3}) b_{1}(4,2,3) a_{1}(\widehat{4}, 1,1) b_{2}(2, \widehat{3}, \widehat{1}) a_{2}(\widehat{2}, 3, \widehat{2}) b_{2}, \\
& v_{2}=(3, \widehat{2}, 4) a_{1}(\widehat{3}, \widehat{4}, \widehat{4}) b_{1} .
\end{aligned}
$$

We obtain $w=\left(w_{1}, \ldots, w_{n}\right) \in \mathscr{W}_{\lambda}$ by retaining in $v=\left(v_{1}, \ldots, v_{n}\right)$ only the $a$ 's and $b$ 's, in place. (Thus we immediately have $\operatorname{wt}(\beta) \operatorname{wt}(\alpha)=\psi(w)$.) We now show that $w$ is in $\mathscr{W}_{\lambda}^{\prime \prime}$. For $i, j \geq 1$, let $\mathscr{E}_{i, j}=\left\{\left(s_{1}, r_{1}, t_{1}\right), \ldots\right.$, $\left.\left(s_{q}, r_{q}, t_{q}\right)\right\}$, where $s_{1} \prec \cdots \prec s_{q}$, be the set of ordered triples $(s, r, t)$ occurring between $a_{i}$ and $b_{j}$ in the $\left(v_{1}, \ldots, v_{n}\right)$, either as a subword $a_{i}(s, r, t) b_{j}$ or $b_{j}(s, r, t) a_{i}$ (circularly).

Consider an arbitrary triple $\left(s_{x}, r_{x}, t_{x}\right)$ in $\mathscr{E}_{i, j}$. From the construction of the $v$ 's, $s_{x}$ is a left-element on a chain labeled $a_{i}$ in $\alpha$, so $r_{x}=\rho\left(s_{x}\right)$ must 
be a right-element on that chain. Similarly, $t_{x}$ is a right-element on a chain labeled $b_{j}$ in $\beta$, so $r_{x}=\sigma^{-1}\left(t_{x}\right)$ is a left-element on that chain. Thus $r_{x}$ appears as a right-element on a chain labeled $a_{i}$ in $\alpha$ and as a left-element on a chain labeled $b_{j}$ in $\beta$ and $s_{x}=\rho^{-1}\left(r_{x}\right), t_{x}=\sigma\left(r_{x}\right)$ can be obtained from $r_{x}$ by means of $\rho$ and $\sigma$. This characterises the triples in $\mathscr{E}_{i, j}$.

Now for such an $r_{x}$, the pair $\left\{r_{x}, \delta\left(r_{x}\right)\right\}$ is a right-link on a chain labeled $a_{i}$ in $\alpha$ and is a left-link on a chain labeled $b_{j}$ in $\beta$. This in turn means that $\delta\left(r_{x}\right)$ is a right-element on a chain labeled $a_{i}$ in $\alpha$ and a left-element on a chain labeled $b_{j}$ in $\beta$, so by the above characterisation, $\delta\left(r_{x}\right)=r_{y}$ for some $\left(s_{y}, r_{y}, t_{y}\right)$ in $\mathscr{E}_{i, j}$, where $y \neq x$. Thus the number of triples, $q$, in $\mathscr{E}_{i, j}$ must be even. Moreover, by construction $q=m_{i, j}(w)+m_{i, j}^{\prime}(w)=\eta_{i, j}(w)$, so $w \in \mathscr{W}_{\lambda}^{\prime \prime}$, with $\lambda=\kappa(\gamma)$.

(iii) For $\mathbf{M}$ : The $(i, j)$-entry of $\mathbf{M}$ is the matching on $\left\{1, \ldots, \eta_{i, j}(w)\right\}$ consisting of all pairs $\{x, y\}$ such that $r_{y}=\delta\left(r_{x}\right)$ in the above analysis.

(iv) For $d$ : Consider the matching $\mathscr{F}$ on $\{1, \hat{1}, \ldots, k, \widehat{k}\}$ consisting of all pairs $\left\{s_{x}, s_{y}\right\}$ such that $r_{y}=\delta\left(r_{x}\right)$ in the above analysis for all $i, j \geq$ 1. Suppose that $\mathscr{F}=\left\{\left\{g_{1}, h_{1}\right\}, \ldots,\left\{g_{k}, h_{k}\right\}\right\}$ where $g_{1} \prec \cdots \prec g_{k}$ and $g_{i} \prec h_{i}, i=1, \ldots, k$. Then $d$ is obtained as $d(i)=\rho\left(g_{i}\right), d(\widehat{i})=\rho\left(h_{i}\right)$, $i=1, \ldots, k$. Clearly, $d \in \mathscr{B}_{k}$ since $\rho\left(g_{i}\right)=\delta\left(\rho\left(h_{i}\right)\right), i=1, \ldots, k$, by construction.

To complete the above example, we obtain

$$
\begin{gathered}
w_{1}=a_{1} b_{1} a_{1} b_{2} a_{2} b_{2}, \\
w_{2}=a_{1} b_{1}, \\
{\left[\mathscr{C}_{i, j}\right]=\left[\begin{array}{cccc}
\{(\widehat{1}, 4, \widehat{3}),(3, \widehat{2}, 4),(\widehat{3}, \widehat{4}, \widehat{4}),(4,2,3)\} & \{(1, \hat{1}, 2),(\widehat{4}, 1,1)\} & \varnothing & \cdots \\
\varnothing & \{(2, \widehat{3}, \widehat{1}),(\widehat{2}, 3, \widehat{2})\} & \varnothing & \cdots \\
\vdots & \vdots & \vdots & \ddots
\end{array}\right],} \\
\mathbf{M}=\left[\begin{array}{cccc}
\{\{1,3\},\{2,4\}\} & \{\{1,2\}\} & \varnothing & \cdots \\
\varnothing & \{\{1,2\}\} & \varnothing & \cdots \\
\vdots & \vdots & \vdots & \ddots
\end{array}\right], \\
\mathscr{F}=\{\{1, \widehat{4}\},\{\hat{1}, \widehat{3}\},\{2, \hat{2}\},\{3,4\}\},
\end{gathered}
$$

and finally

$$
d=\left(\begin{array}{llllllll}
1 & \hat{1} & 2 & \widehat{2} & 3 & \widehat{3} & 4 & \widehat{4} \\
\hat{1} & 1 & 4 & \widehat{4} & \widehat{3} & 3 & \widehat{2} & 2
\end{array}\right) .
$$

To reverse the construction, we start with $\gamma, w, \mathbf{M}, d$ and proceed to determine $v$. The second and fourth position $(\bmod 4)$ of $v$ come immediately from $w$. The first and third entries in the triples occupying the first and third positions $(\bmod 4)$ of $v$ come immediately from the chain decomposition of $\gamma$. These give the first and third entries of the triples in $\mathscr{E}_{i, j}$ for each $i, j \geq 1$, depending between which $a_{i}$ and $b_{j}$ they lie. This portion of the $\mathscr{E}_{i, j}$ together with $\mathbf{M}$ give the matching $\mathscr{F}$. Finally $d$ and $\mathscr{F}$ together allow us to determine the second entries of the triples in $\mathscr{E}_{i, j}$ and thence recover $v$. Clearly, $v$ uniquely determines $\rho, \sigma, \alpha, \beta$. 


\section{REFERENCES}

1. I. P. Goulden and D. M. Jackson, The combinatorial relationship between trees, cacti and certain connection coefficients for the symmetric group, European J. Combin. 13 (1992), 357-365.

2. P. J. Hanlon, R. P. Stanley, and J. R. Stembridge, Some combinatorial aspects of the spectra of normally distributed random matrices, Contemp. Math., vol. 138, Amer. Math. Soc., Providence, RI, 1992, 151-174.

Department of Combinatorics \& Optimization, University of Waterloo, Waterloo, Ontario, Canada N2L 3G1

E-mail address: ipgoulden@math.uwaterloo.ca

E-mail address: dmj ackson@dragon.uwaterloo.ca 